\title{
Informatik in der Medizin
}

$\mathrm{D}$ ie aktuelle Rolle der Informationstechnologie (IT) im Krankenhaus ist durch Licht und Schatten gleichermaßen geprägt: einerseits sind Datenverarbeitungssysteme bahnbrechende Werkzeuge zur Unterstützung klinischer und institutionsübergreifender Prozessketten, andererseits lediglich Hilfsinstrumente für eine ausufernde administrativ geprägte Dokumentationspflicht.

Es gibt Stimmen, welche die Informationstechnologie im Krankenhaus insgesamt kritisch beurteilen. So meinte das amerikanische „Institute of Medicine“ im Jahr 2001, dass die Revolution der Informationstechnologie, die in Industrie und Wirtschaft zu dramatischen Veränderungen geführt hat, an der Medizin nahezu spurlos vorübergegangen ist. Erfahrungsberichte bestätigen, dass IT-Lösungen im Krankenhaus personalaufwändig sind und dass klinische IT-Projekte unter verschiedensten Schwachstellen leiden, zu denen insbesondere zeitliche Verzögerungen und enttäuschte Erwartungen zählen. Die bereits seit den 80er Jahren thematisierte Software-Krise ist noch nicht überwunden. Auch die angesichts der Umwälzungen durch die DRG-Einführung häufig geforderte IT-Unterstützung klinischer Arbeitsprozesse ist heute noch wenig ausgeprägt - dies gilt abteilungsintern und umso mehr abteilungs- oder gar institutionsübergreifend. Das erklärte Ziel, Wissen und Information am Punkt der Krankenversorgung umfassend und zeitnah zur Verfügung zu stellen, ist nur in Ansätzen erreicht.

Das Potenzial der Informationstechnologie zur Unterstützung des Klinikers wird dagegen immer besser verstanden. Eine geeignete Arbeitsumgebung, die dem Arzt und dem Pflegepersonal relevante und angemessen aufbereitete Information über den Patienten und den Behandlungsprozess zur Verfügung stellt, könnte die hohe kognitive Belastung reduzieren. IT-basierte Erinnerungshinweise können helfen, Unterlassungsfehler zu vermeiden, und Warnhinweise können Fehler verhindern, die aus Übersehen oder Vergessen (etwa von Nebenwirkungen oder Kontraindikationen) oder auch aus der Unterlassung notwendiger Kontrollmaßnahmen resultieren. Darüber hinaus hat die Telematik ihre Kinderschuhe verlassen und könnte dazu beitragen, die Kommunikation zwischen Krankenhaus und Niedergelassenen zu unterstützen.

In der Realität ist die Entlastung durch die Informationstechnologie bisher kaum wahrzunehmen. Während der Druck auf Krankenhausärzte wächst, wird IT vielfach vorrangig eingesetzt, um gesetzliche Dokumentationsanforderungen umzusetzen. Der jüngst vom Deutschen Krankenhausinstitut ermittelte Zeitaufwand für die Dokumentation im Krankenhaus lag bei über zwei Stunden für patientenbezogene und bei rund 40 Minuten für administrative Tätigkeiten, zum Beispiel das Erfassen von ICD- und OPS-Codes. IT imponiert somit häufig primär als Werkzeug zur Bewältigung eines wachsenden Dokumentationsaufwands, der zeitlich mit der primären Patientenversorgung konkurriert. Erschwerend kommt hinzu, dass der Mitteleinsatz für die klinische Informationsverarbeitung in Deutschland eher gering ist und bei maximal 1-2\% des Budgets im Vergleich zu mindestens $2-3 \%$ in den USA liegen dürfte.

Begrenzte Mittel müssen also gezielt zur IT-Unterstützung des Behandlungsprozesses eingesetzt werden. Neben einem Ausbau der IT-Infrastruktur im Krankenhaus bedeutet dies Maßnahmen zur Unterstützung der Kooperation und der zeitnahen Information. Zur Verbesserung der Koordination gewinnen Terminplanung und elektronische Auftragsübermittlung an Bedeutung, die in immer breiteren Fassetten auch abteilungsübergreifend eingesetzt werden. Von herausragender Bedeutung ist eine Fokussierung auf die Durchgängigkeit der Prozessketten. Hersteller wie auch Anwender werden ihr Augenmerk zunehmend hierauf richten müssen.

In diesem Kontext geht die Wissensvermittlung neue Wege. Fakten und Wissensbanken im Internet und im Intranet sind bereits heute dabei, einen erheblichen Einfluss auf die klinische Routine zu gewinnen. Darüber hinaus beginnen sich Rolle und Erscheinungsbild der ITunterstützten Entscheidungsunterstützung zu wandeln. Klinische Pfade, die sowohl klinische als auch ökonomische Gesichtspunkte berücksichtigen, können nicht nur im Intranet präsentiert und aus dem Informationssystem aufgerufen werden, sie werden auch zunehmend in klinische Informationssysteme abgebildet werden. Hier zeichnet sich eine problemorientierte Dokumentation mit Fokussierung auf Entscheidungsschritte von klinischer und ökonomischer Relevanz ab. Auch im Bereich der Bild- und Biosignalverarbeitung nimmt die Bedeutung der Informationstechnologie stetig zu.

Insgesamt ist zu hoffen und zu fordern, dass sich die Informationstechnologie von einem momentan stark administrativ geprägten Fokus wieder dem klinischen Prozess und der Entlastung des Klinikers zuwendet.

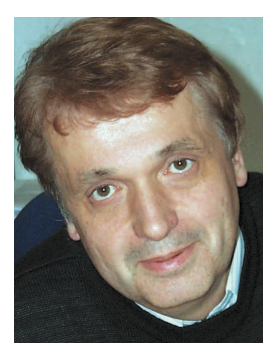

Prof. Dr. K.A. Kuhn, Marburg

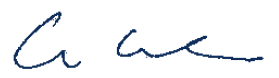

